\title{
EFFECT OF RIGHT CENSORING BIAS ON SURVIVAL ANALYSIS
}

A PREPRINT

\author{
Enrique Barrajón \\ Department of Medical Oncology \\ Hospital General Universitario de Elche \\ Spain \\ enrique.barrajon@gmail.com
}

\author{
Laura Barrajón \\ Department of Hospital Pharmacy \\ Hospital Universitario San Juan de Alicante \\ Spain
}

December 17, 2020

\begin{abstract}
Kaplan-Meier survival analysis represents the most objective measure of treatment efficacy in oncology, though subjected to potential bias, which is worrisome in an era of precision medicine. Independent of the bias inherent to the design and execution of clinical trials, bias may be the result of patient censoring, or incomplete observation. Unlike disease/progression free survival, overall survival is based on a well defined time point and thus avoids interval censoring, but right-censoring, due to incomplete follow-up, may still be a source of bias. We study three mechanisms of right-censoring and find that one of them, surrogate of patient lost to follow-up, is able to impact Kaplan-Meier survival, improving significantly the estimation of survival in comparison with complete follow-up datasets, as measured by the hazard ratio. We also present two bias indexes able to signal datasets with right-censoring associated overestimation of survival. These bias indexes can detect bias in public available datasets.
\end{abstract}

Keywords clinical trials $\cdot$ missing data $\cdot$ reproducibility of results $\cdot$ right-censored outcome $\cdot$ statistical bias $\cdot$ survival analysis

\section{Introduction}

More than one hundred years after the statement that certainty in science requires the confirmation of insights by measurement [1], the scientific community is acknowledging a reproducibility crisis in the last few years [2].

Clinical trials represent an area of biomedical research affected by the replication crisis. About one third of randomized clinical trials subjected to reanalyses have shown different conclusions to the original article [3], and around 10\% of trial conclusions reported to the Food and Drug Administration (FDA) show some kind of discordance to the published articles [4], and, for this reason, it has been claimed that most of research resources are wasted for this reason [5]. It is worrisome that, in an age of precision medicine, clinical research is threatened by different kinds of biases inherent to the design, implementation, and analysis of clinical trials, compromising the translation of trial outcomes into clinical benefit [6]. It has been postulated that even a totally unbiased, perfectly designed and executed clinical trial may still produce false and irreproducible results [7].

Survival analysis, as described by Kaplan and Meier, extract the most information of incomplete observations (censoring) and represents the most objective measure of treatment efficacy in oncology, medical sciences in general, and engineering [8]. Censoring means the survival time to event for a subject cannot be accurately determined because dropout, lost to follow-up, unavailable information, or the study ends before the event of interest occurs, and may affect the estimation of survival rate [9].

To estimate survival as a function of time, Kaplan-Meier analysis arranges all the $n$ study cases, sorted by the time of follow-up, giving $n$ intervals. Starting with $S_{t i m e=0}=1$, the probability of survival is recalculated at every event, with the product of the probability of survival at the previous interval and the survival ratio in the interval: surviving patients 
at the end of the interval divided by the number of patients that entered this interval (the probability of survival is not updated at censoring times.)

$$
S_{i}=\prod_{i=1}^{n}\left(1-\frac{\text { events }_{i}}{\text { atRisk }_{i}}\right)
$$

While almost half of Kaplan-Meier analyses published in medical journals are susceptible to competing risk bias that may overestimate event risk [10], informative censoring bias may be associated with overestimation of treatment effects [11]. Censoring may change the conclusions of clinical trials and may challenge the validity of results from interim analyses in clinical trials [12, 13].

One of the problems with survival analysis is the accuracy of the start and event times, which may give rise to interval censoring [14]. This uncertainty is reduced in clinical trials with a perfect definition of the patient entry and event dates, but, sometimes, the event time only can be assigned within an interval, as when we measure disease free survival or progression free survival. Overall survival, otherwise, is based on a well defined time point and, thus, avoids interval censoring, but it may still be the subject of right-censoring when we do not know the event time point. Another drawback of estimating overall survival is that more patients and time are needed, and others factors, or further treatments, may affect outcomes [15, 16, 17, 18].

The Kaplan Meier survival curve, as well as the logrank test, are based on the assumptions that censoring is unrelated to prognosis (the random censoring model), the survival probability is independent of the chronological time of inclusion in the study, and events happen at the times specified. Deviations from these assumptions may affect results if they are satisfied differently in the groups being compared, for example if censoring is more likely in one group than another [19]. It has been stated that elimination from the database of patients lost to follow-up or who withdraw consent may affect the power and validity of conclusions derived from the clinical study [20]. Validity of the random censorship model, that we take for granted, may not be proved nor excluded with a statistical test, and might lead to biased results of the Kaplan-Meier estimator [21].

In this project, the $R$ language [22], the integrated development environment RStudio [23], the survival [24], and OptimalCutpoints [25] packages were used to simulate population time to event, censoring time, and to the systematic study of bias, as well as the potential bias in public available datasets [26].

\section{Virtual datasetes}

The survival package uses datasets with, at least, a vector of times, and a vector of states, the latter informing the state of every case: event or censored. To study the effect of censoring on survival, we simulate datasets with complete follow-up and derive from them datasets with different patterns of censoring.

\subsection{Complete follow-up}

The survival function in patients with cancer, or any other condition, may be simulated according to a Weibull mixture cure-rate model, where the survival probability is the result of a subpopulation of cases with events (deaths, relapses, etc.) occurring following a Weibull distribution, plus a subpopulation of cured cases, never experiencing the event [27]. The cured fraction has been defined as the proportion of patients with the same death rates of the general population. A problem with this definition is that the time when the death rate is similar to the general population differs by tumor type, and is much longer than the usual duration of studies, and the normal approach is to talk about the proportion of long-term survival (at some arbitrary time interval) [28].

A hypothetical dataset with complete follow-up may be approached by a general 2-parameter Weibull reliability/survivorship function with shape parameter $k \in(0, \infty)$ and scale parameter $b \in(0, \infty)$ [29]. The exponential function is a particular case of the Weibull function with $k=1$.

$$
S(t)=e^{-\left(\frac{t}{b}\right)^{k}}
$$

The inverse Weibull distribution allows to simulate a time distribution $t$ knowing the parameters $b$ and $k$, and a random probability of survival $p \in(0,1)$.

$$
t=b\left(\ln \frac{1}{p}\right)^{1 / k}
$$


Parameters $k$ and $b$ have no practical meaning, and a more convenient way of representing the 2-parameter Weibull survival function is as a function of quantiles, which can be measured directly in survival datasets. The quantiles in the Weibull cumulative distribution are defined by the expression:

$$
Q_{p}=b[-\ln (1-p)]^{1 / k}
$$

We may use this expression with two known quantiles, such as the median, $Q_{50}$, and the 99th percentile, $Q_{99}$,

$$
\begin{gathered}
Q_{50}=b(\ln 2)^{1 / k} \\
Q_{99}=b(\ln 100)^{1 / k}
\end{gathered}
$$

to obtain the parameters shape $k$ and scale $b$ of a Weibull distribution:

$$
\begin{gathered}
k=\frac{\ln (\ln 100-\ln 2)}{\ln Q_{99}-\ln Q_{50}} \\
b=\frac{Q_{50}}{(\ln 2)^{1 / k}}
\end{gathered}
$$

Using these tools, we may obtain a dataset with vector of times and states. The vector of times is obtained from a vector of random probabilities and the parameters of a Weibull mixture cure-rate distribution: the median event time, the 99th percentile event time, and a given cure-rate for the population. For convenience, the 99th percentile event time in the virtual datasets is fixed to 100 units of time and we let the median survival vary in the range 5-95 units of time; the cure rate is simulated in the arbitrary range $0-80 \%$. For a complete follow-up dataset, the states vector is 0 for the cured cases, and 1 all the other cases, event cases.

\subsection{Incomplete follow-up}

We may obtain censored datasets from a complete follow-up dataset by one of three different mechanisms of censoring:

\section{A. Time censoring}

For every case in the complete follow-up dataset, a new random time is generated from a Weibull distribution with median and $Q_{99}$ percentile times, and a proportion of censoring. Every case is updated if this new time is shorter than the complete follow-up time: the new time replaces the complete follow-up one and the state is updated to 0 (censored).

\section{B. Interim censoring}

A random interim time is obtained from a Weibull distribution with median and $Q_{99}$ percentile times. For every case in the complete follow-up dataset, a random recruit time is generated. If the interval between recruit time and interim time is shorter than the complete follow-up time, this interval replaces the complete follow-up time and the state is changed to 0 (censored). This mechanism parallels the censoring associated with interim analysis.

\section{Case censoring}

A random sample of cases is selected from the complete follow-up dataset with a given probability of censoring. For these censored cases, the corresponding time is shortened a random amount, and the corresponding state is changed to 0 (censored). This mechanism of censoring assimilates retired cases from clinical trials, either lost to follow-up or who withdraw consent.

\section{Virtual trials}

A virtual trial is constituted by a censored dataset and the corresponding complete follow-up dataset. The complete follow-up dataset is simulated with one thousand cases following a Weibull mixture cure-rate model. The censored dataset is obtained from the complete follow-up dataset, applying one of the three described mechanisms of censoring. 
The virtual trial allows to study the impact of censoring on survival, by estimating the hazard ratio $(H R)$ and $p$-value of the censored dataset compared with the complete follow-up dataset. A hazard ratio of 1 means that the hazard of the censored dataset and the complete follow-up dataset are the same, while a hazard ratio less than 1 means the hazard of the censored dataset is less than the hazard of the complete follow-up dataset; a p-value less than 0.05 means a low probability that the results are due to chance [30]. The complete follow-up and censored Kaplan-Meier survival curves may be represented together.
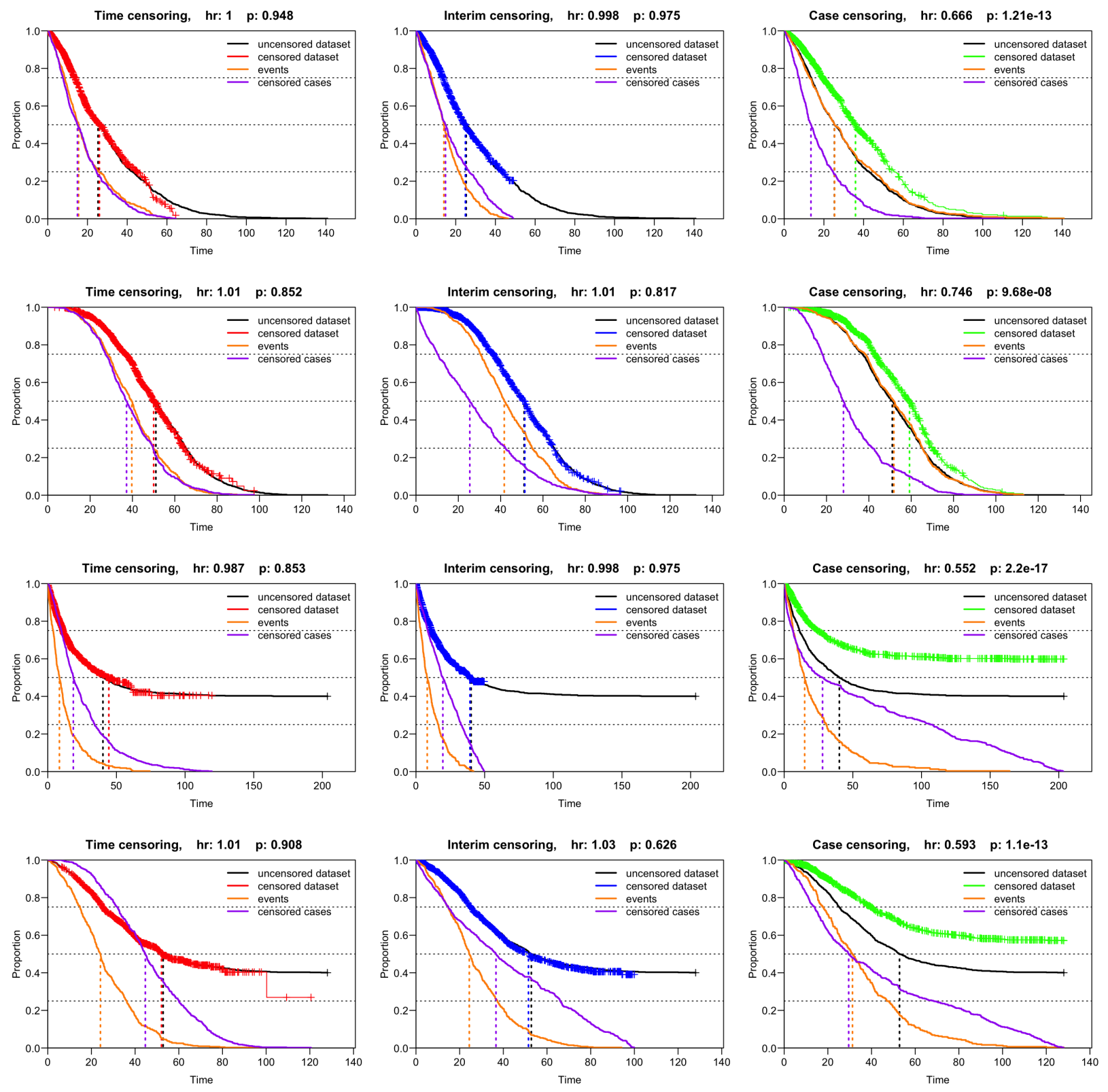

Figure 1: Influence of censoring type on Kaplan-Meier estimate (see text)

Figure 1 shows 12 virtual experiments based on 4 complete follow-up datasets with median event time of 25 and 50 time units ( $Q_{99}$ percentil of 100 time units), and cure-rate of 0 and 0.4 . For every complete follow-up dataset, three censored datasets are obtained with the three different mechanisms of censoring and proportion of censoring of 50\%. For every virtual trial, we plot the complete follow-up dataset in black, and the censored dataset in color: red for time censoring, blue for interim censoring, and green for case censoring. In addition, for the censored dataset, we represent 
differentially the survival curves of event $(\mathcal{E})$ and censored $(\mathcal{C})$ cases in the events interval $t \in(0, \max (\mathcal{E}))$ : event times in orange, and censored times in purple, to find out patterns that may be associated with a potential change in survival.

While time censoring and interim censoring survival curves overlap with the complete follow-up survival curve, with HR around 1, case censoring shows an increase in survival signaled by HR below 1. An interactive simulation is presented at https://adio.shinyapps.io/right_censoring.

\section{Virtual experiments}

Virtual experiments are composed of hundreds of virtual trials. In a typical virtual experiment, 1000 random complete follow-up datasets of 1000 cases are simulated with a fixed $Q_{99}$ percentil of event times of 100 units and random values of median event time in the range 5 to 95 units, and random cure-rate in the range 0-0.8. For every complete follow-up dataset, three censored datasets are simulated with each of the described mechanisms of censoring and random proportion of censoring in the range of 5\% to $95 \%$, resulting in 3000 virtual trials. Every virtual trial may be represented as a dot in a scatter plot: time censoring (red), interim censoring (blue) and case censoring (green). Figure 2 shows a scatter plot of HR vs the proportion of censoring; solid dots represent trials with a significant change in HR (p-value $<0.05)$. We may observe how, unlike time censoring and interim censoring, case censoring is associated with a biased estimation of survival: HR correlates inversely with the proportion of censoring $(r=-0.939)$.

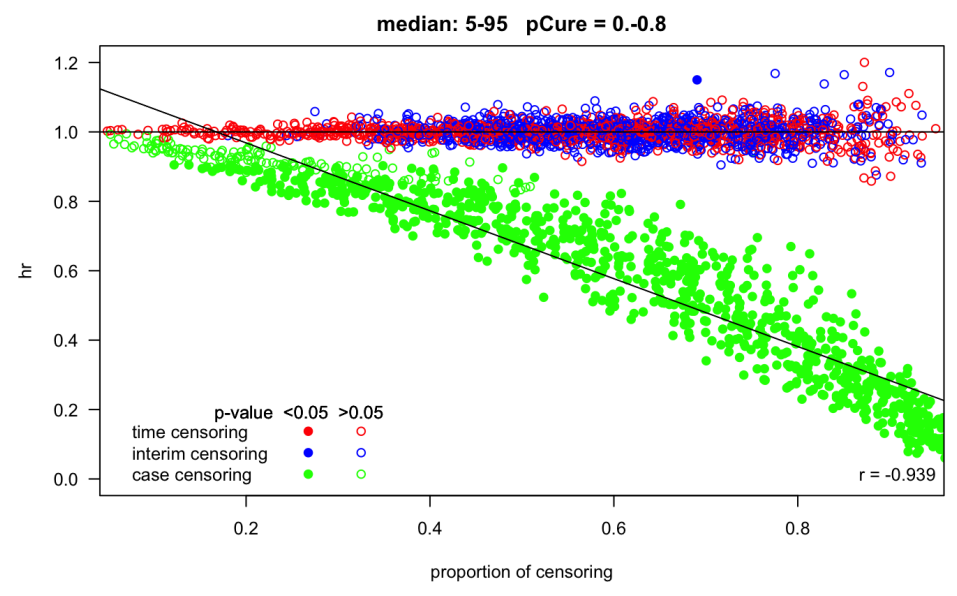

Figure 2: Influence of censoring proportion on survival estimation

\subsection{Bias measures}

To study bias, virtual experiments were simulated with thousands of virtual trials with random Weibull mixture cure-rate models. For every virtual trial, we estimate the HR and p-value of the censored to complete follow-up datasets, and a bias index (BI). A pragmatic approach was used to find a BI able to signal virtual trials decreasing the HR with a statistically significant p-value. Scatter plots of HR vs BI represent every virtual trial as a dot (trials based on time censoring in red, interim censoring in blue, and case censoring in green; solid circles denote p-value < 0.05) [Fig. 3].

In order to obtain a cutoff for the BI to maximize the sensitivity and specificity to classify virtual trials as biased (p-value $<0.5$ ) or unbiased, the Receiver Operating Characteristic (ROC) and the Youden index were estimated with the OptimalCutpoints package lcite\{Lopez2014]. The obtained cutoff is then used to rescale the BI to obtain a scaled BI with cutoff of 1 , a convenient threshold indicating that the virtual trial may be biased.

\subsubsection{Quantile bias index (QBI)}

Biological distributions, and survival distributions in particular, are frequently right-skewed, as common measures, such as time, have a lower bound of zero [31]. A first glance at right skewed virtual trials of figure 1 shows that the observed significant decrease in HR found in case censoring trials (green curves) might be associated with the separation of events and censored subgroups of the censored dataset in the right-most part of their survival curves. A QBI function was constructed based on the ratio of 95th quantiles of event times and censor times: 


$$
Q B I=\frac{Q_{0.95}(\mathcal{E})}{Q_{0.95}(\mathcal{C})}
$$
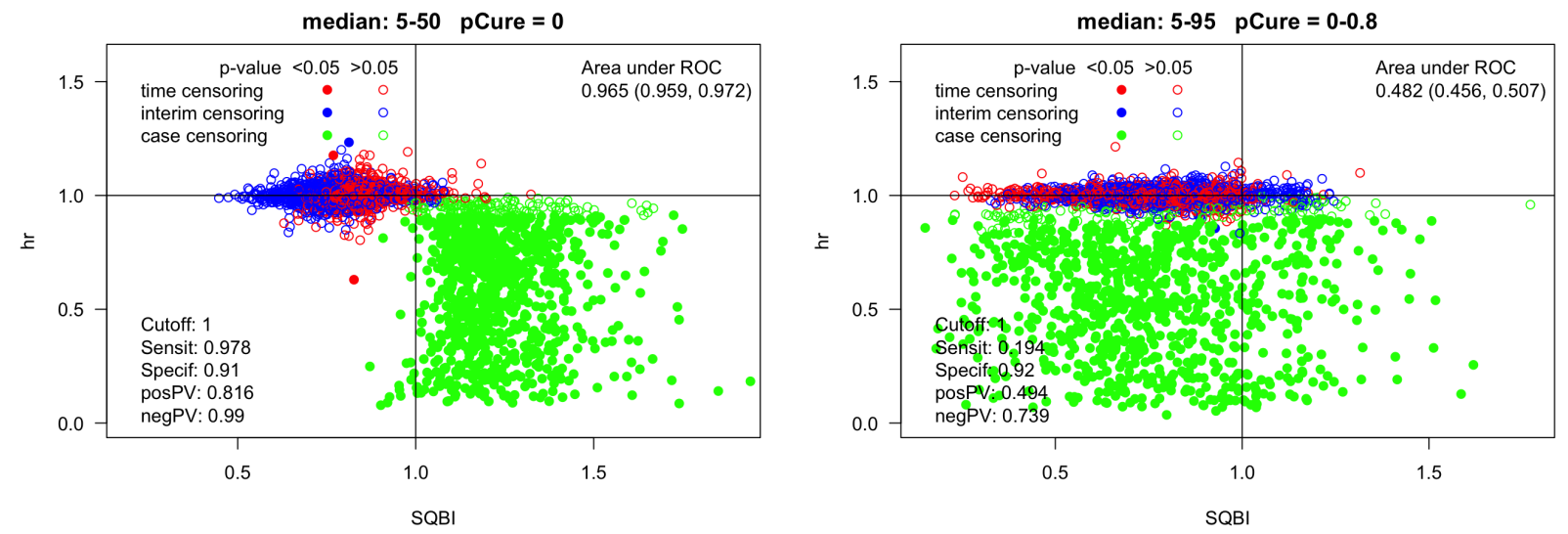

Figure 3: Influence of SQBI on survival estimation for right skewed distributions with cure-rate $=0$ (left panel) or overall (right panel)

Figure 3 shows 2 experiments, each of them with 3000 trials based on 1000 complete follow-up datasets and the 3 mechanisms of censoring described, with random proportion of censoring in the range 0.05 to 0.95 . QBI differentiates pretty well for right skewed distributions (datasets with median event time below 50 unit times) and cure-rate of 0 (figure 3 left panel). A QBI cutoff of 1.2 separates efficiently virtual trials with a significant decrease in HR from virtual trials with HR around 1, with an area under the Receiver Operating Characteristic (ROC) curve of 0.965, and 95\% confidence interval (CI) of 0.959-0.972. A scaled QBI (SQBI) is calculated dividing the QBI by the cutoff found:

$$
S Q B I=\frac{Q B I}{\text { cutoff }}
$$

SQBI, nevertheless, fails with trials based on symmetric or left skewed distributions of events, or when there are a proportion of long-term survival cases. The estimated area under the ROC curve is 0.49 (95\% CI: $0.465-0.515$ ) for trials with median event times in the range of 5-95 units and cure-rate of 0-80\% (figure 3 right panel), equivalent to diagnose bias by pure chance.

\subsubsection{Under the mean bias index (UMBI)}

A more consistent $\mathrm{BI}$ is based on the proportion of censored cases in the event interval, $t \in(0, \max (\mathcal{E}))$, which are under the mean event time.

$$
U M B I=\frac{|\mathcal{C}<\overline{\mathcal{E}}|}{|\mathcal{C}<\max (\mathcal{E})|}
$$

Figure 4 represents 4 virtual experiments with a $2 \times 2$ design: right skewed Weibull distributions or not, and cure-rate of 0 or $50 \%$. UMBI separates virtual trials with a significant decrease in HR from virtual trials with HR around 1 , with area under the ROC curve over 0.703 ( 0.961 for right skewed distributions and no cure-rate), but the cutoff is not consistent: it increases with the median event time and decreases with the proportion of long term survivors. Thus, we need to normalize this index to get a consistent index independent of median event time and cure-rate. Since the cure-rate may be unreliable to be estimated from an incomplete follow-up dataset, the long-term proportion of cases after the last event is used. 

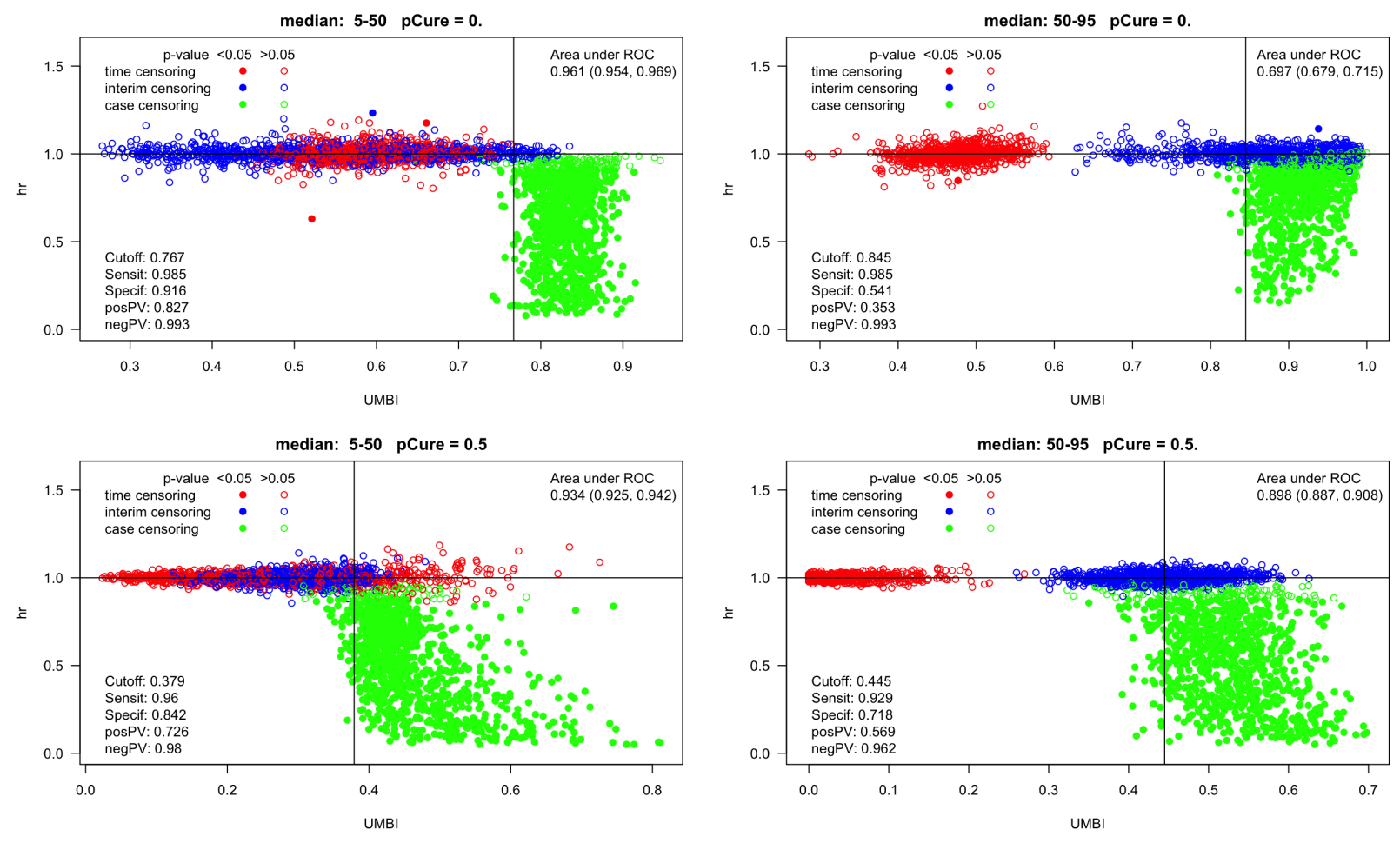

Figure 4: Influence of UMBI on survival estimation

\subsubsection{Adjusted bias index (ABI)}

To normalize UMBI, we need to adjust for survival skewness and the proportion of long-term survival cases, multiplying by two factors to obtain the pursued ABI:

- Multiplying by the ratio of mean $\overline{\mathcal{C}}$ to median $\widetilde{\mathcal{C}}$ censored times, corrects for the effect of skewness in the distribution.

- Multiplying by $e$ to the power of the estimator of Kaplan-Meier long-term survival $\widehat{S}_{K M(t=\max (\mathcal{E}))}$ corrects for the effect of the long-term survival rate.

$$
A B I=\frac{|\mathcal{C}<\overline{\mathcal{E}}|}{|\mathcal{C}<\max (\mathcal{E})|} \frac{\overline{\mathcal{C}}}{\widetilde{\mathcal{C}}} e^{\widehat{S}_{K M(t=\max (\mathcal{E})) S}}
$$

After these adjustments, the $\mathrm{ABI}$ cutoff for trials based on median event times of 5 to 50 units and cure-rate of 0 is 0.909 , with an area under the ROC curve of 0.822 (95\% CI 0.808-836), worse than for the QBI. However, as can ben seen in figure 5. for datasets based on median event times of 5-95 units and cure rate of $0-80 \%$, the cutoff is 0.932 , and the area under the ROC curve increases to 0.91 (95\% CI: 0.899-0.921). A scaled ABI (SABI) is calculated dividing the ABI by the cutoff:

$$
S A B I=\frac{A B I}{\text { cutoff }}
$$

Thus, both scaled indexes, SQBI and SABI, appear effective to detect bias in the survival analysis of clinical trials and are supplementary. SQBI appears superior to SABI for right skewed Weibull distributions with cure rate of 0\%, with a high sensitivity of 0.978 and specificity of 0.91 . For all the remained cases, SABI is better, with and accceptable sensitivity of 0.866 and specificity of 0.842 . 

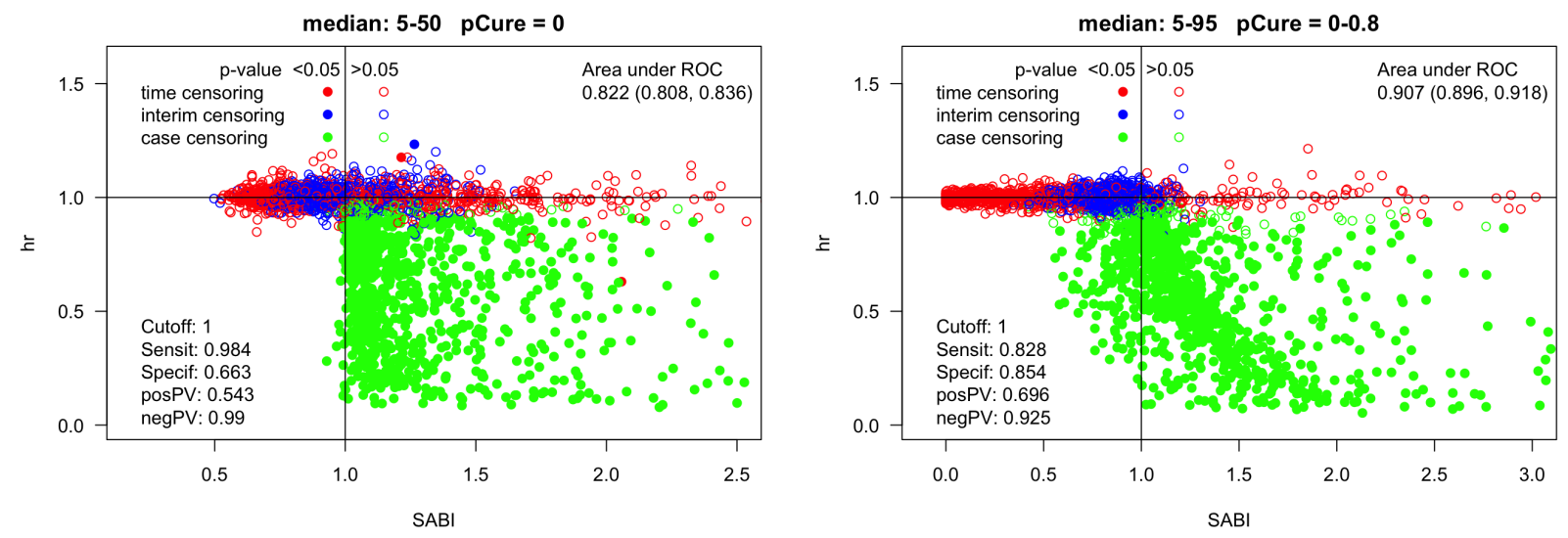

Figure 5: Influence of SABI on survival estimation for right skewed distributions with cure-rate $=0$ (left panel) or overall (right panel)

\section{Clinical available datasets}

Available clinical datasets were retrieved from Documentation for package survival version 2.44-1.1, based on 6 published articles [26]. Bias indexes SQBI and SABI were applied to the datasets and to the research arms where available.

Table 1: Bias in available clinical datasests

\begin{tabular}{lrrlll}
\hline trial & $\mathrm{n}$ & $\mathrm{pCens}$ & SQBI & SABI & reference \\
\hline Acute Myelogenous Leukemia survival data & 23 & 0.22 & 0.89 & 0.58 & {$[32]$} \\
Bladder Cancer Recurrences - bladder1 & 294 & 0.35 & 0.51 & 0.69 & {$[33]$} \\
- placebo & 128 & 0.31 & 0.66 & 0.61 & \\
- pyridoxine & 85 & 0.33 & 0.3 & 0.7 & \\
- thiotepa & 81 & 0.43 & 0.85 & $\mathbf{1 . 3}$ & \\
Bladder Cancer Recurrences - bladder2 & 178 & 0.37 & 0.6 & 0.78 & {$[33]$} \\
- rx1 & 106 & 0.32 & 0.69 & 0.8 & \\
- rx2 & 72 & 0.44 & 0.87 & 0.92 & \\
NCCTG Lung Cancer Data & 228 & 0.28 & 0.79 & 0.73 & {$[34]$} \\
Chemotherapy for Stage B/C colon cancer & 1858 & 0.50 & 0.59 & 0.04 & {$[35]$} \\
- Observation & 630 & 0.45 & 0.62 & 0.048 & \\
- Levamisol & 620 & 0.46 & 0.57 & 0.037 & \\
- Levamisol + 5FU & 608 & 0.60 & 0.62 & 0.041 & \\
Ovarian Cancer Survival Data & 26 & 0.54 & $\mathbf{1 . 1}$ & 0 & {$[36]$} \\
- rx1 & 13 & 0.46 & 1 & 0 & \\
- rx2 & 13 & 0.62 & $\mathbf{1 . 1}$ & $\mathbf{1 . 8}$ & \\
Veterans' Administration Lung Cancer Study & 137 & 0.07 & $\mathbf{1 . 5}$ & $\mathbf{1 . 4}$ & {$[37]$} \\
- standard & 69 & 0.07 & $\mathbf{1 . 8}$ & 0.8 & \\
- test & 68 & 0.06 & $\mathbf{1 . 8}$ & $\mathbf{2}$ & \\
\hline
\end{tabular}

A SQBI over 1 was found in the Veterans' Administration Lung Cancer Study overall, as well as the standard and test arms, suggesting right-censoring bias. The Veterans' Administration Lung Cancer Study has a righ skewed distribution of times and a long-term survival rate of 0, giving strength to this result as a true positive [37]. The SQBI of 1.1 found in the Ovarian Cancer Survival Data overall and in the rx 2 treatment arm appears confirmed in the rx 2 arm by a SABI of 1.8, but bias may be the result of the small number of cases [36]. The isolated SABI of 1.3 for the thiotepa arm of the Bladder Cancer Recurrences dataset may alert us about the presence of bias, suggesting the need for updating results. 


\section{Discussion}

In this study, we have addressed the problem of the potential bias associated with right-censoring in clinical trials, a technical problem beyond the common biases associated with research design and execution, because it is inherent to the methodology of Kaplan-Meier survival analysis and may be the source of bias even in spite of a well designed and executed trial. We developed three different mechanisms of right-censoring: time censoring, interim censoring and case censoring, based on common simulation censoring models, the well known interim analysis performed before a clinical trial has been completed, and the loss of information because of patients lost to follow-up or withdraw of consent, respectively. It is shown that one of the three mechanisms of censoring, case censoring, equivalent to dropouts and lost to follow-up, is associated with a biased improvement of results compared with complete follow-up, as demonstrated by a decrease in the HR of censored group to complete follow-up group below 1 and a p-value less than 0.05 . This impact on HR is inversely correlated to the proportion of censored cases. Interim censoring, where entry in the study is random and censoring date is the date of interim analysis or end of the study, guarantees that censoring and event times are independent [21].

Though case censoring may be considered a hypothetical factor in clinical trials, a systematic review has acknowledged a median proportion of patients lost to follow-up of $6 \%$ (when reported, as $13 \%$ of trials do not give this information at all). Different assumptions about what proportion of cases lost to follow-up end having the event may change the results in up to a third of trials [38]. The most extreme case is the estimated fourfold overestimation of survival in patients with a resected pancreatic cancer which have been attributed to a $60 \%$ of patients lost to follow-up [39]. A systematic review of clinical trials on antiretroviral therapy in low and middle income countries recognized that a large proportion of patients are lost to follow-up. Increasing physical tracing is associated with a lower proportion of patients lost to follow-up (7.6\% vs. $15.1 \%$; $<<0.001)$ and higher estimated mortality $(10.5 \%$ vs. $6.6 \% ; \mathrm{p}=0.006)$, in spite of higher retention on treatment $(80.0 \%$ vs. $72.9 \% ; \mathrm{p}=0.02)$, suggesting an overestimation of survival associated with censoring [40]. In the same way, the Kaplan-Meier survival analysis of patients with squamous cell carcinoma of head and neck reveals that updating the information of patients lost to follow-up anytime, decreases significantly the 10 years survival estimation $(28.2 \%$ vs $46 \%, \mathrm{p}<0.001)$ [41]. The theoretical framework we present here is consistent with all those studies.

A first solution to the problem of bias associated with incomplete follow-up is to maximize event information. Even though patient participation in a clinical trial may be stopped, at any time, by patient or clinician decision, withdrawn consent for treatment does not imply withdrawn consent for obtaining information. It should be imperative for patients, clinicians and researchers to maintain the loss of information to a minimum, though data on morbidity may be more difficult to obtain than mortality data in these patients [20]. In addition, the censoring proportion should be reported in the control and experiental arms of clinical trials, as systematic bias may influence results [42].

The two presented bias indexes, SQBI and SABI, may suggest the presence of bias and help intensify the efforts to obtain information. In serious diseases where the event is the norm in a short time interval, such as advanced pancreatic cancer, SQBI may be appropriate; in more indolent diseases with long-term survival rate, as the case of adjuvant treatment in breast cancer patients, SABI may be more appropriate. Aggressive neoplasms with some long-term survivors, as recent trials of advanced lung cancer on immunotherapy, represent a challenge for both BI. The negative predictive values are very high: 0.99 for SQBI and 0.939 for SABI, suggesting that a BI less than 1 is reassuring. Unfortunately, the positive predictive values are lower: 0.816 for SQBI and 0.69 por SABI, and both indexes should be used as a warning of bias, as they are associated with $20-30 \%$ of false positives. Thus, a SQBI and/or SABI greater than 1 should warn about potential bias and indicate the need of updating results and replication. Replication of clinical trials results using other trials or real-world evidence should be pursued to advance certainty and regulatory science [43].

It has been claimed that survival data may be viewed as the competition of three risks: risc of failure/event, administrative censoring, both mutually independent, and dropout/loss to follow-up censoring, which may not be independent of failure risk [21]. A strategy to attenuate right-censoring bias could be to differentiate lost to follow-up from other kinds of censoring, and treat them differently in the Kaplan-Meier analysis, such as assigning them a certain risk of event in the interval, interpolated from the previous and following event cases, or modifying its follow-up time by a correction factor.

The bias detected in the Veterans' Administration Lung Cancer Study are proof of concept that right-censoring bias in clinical trials may be detected and measured. It would have been very helpful to update the survival time of censored cases to know if these results hold, as has been reported in the forementioned squamous cell carcinoma of head and neck trial [41]. Censoring bias analysis should be pursued in clinical trials. A censoring bias analysis in the public platform projectdatasphere.org is under way. 


\section{Acknowledgements}

The authors thank Dr. A. López, for his constructive criticism and discussion on this subject, and to the people that create and maintain open-source software packages we use.

\section{Conflict of interest}

None

\section{Data availability}

The data and model code on which these results are based will be available in the APPENDIX, at the end of this article.

\section{ORCID}

Enrique Barrajón https://orcid.org/0000-0002-6648-5704

Laura Barrajón https://orcid.org/0000-0002-2004-3708

\section{References}

[1] Arno Laesecke. Through measurement to knowledge: The inaugural lecture of heike kamerlingh onnes (1882). Journal of Research of the National Institute of Standards and Technology, 107:261-277, 2002. doi: 10.6028/jres.107.021.

[2] Monya Baker. 1,500 scientists lift the lid on reproducibility. Nature, 533(425):452-454, 2016. $10.1038 / 533452 \mathrm{a}$

[3] Shanil Ebrahim, Z.N. Sohani, Luis Montoya, Arnav Agarwal, Kristian Thorlund, Edward J. Mills, and John P. A. Ioannidis. Reanalyses of randomized clinical trial data. JAMA, 312(10):1024-1032, 2014. doi: 10.1001/jama.2014.9646.

[4] Kristin Rising, Peter Bacchetti, and Lisa Bero. Reporting bias in drug trials submitted to the food and drug administration: Review of publication and presentation. PLoS Medicine, 5(11):1561-1570, 2008. doi: 10.1371/journal.pmed.0050217

[5] John P.A. Ioannidis. How to make more published research true. PLoS Medicine, 11(10):1001747, 2014. doi: 10.1371/journal.pmed.1001747.

[6] Carl Heneghan, Ben Goldacre, and Kamal R. Mahtani. Why clinical trial outcomes fail to translate into benefits for patients. Trials, 18(1):122, Mar 2017. doi: 10.1186/s13063-017-1870-2.

[7] Leonid Hanin. Why statistical inference from clinical trials is likely to generate false and irreproducible results. BMC Med Res Methodol, 17:127, 2017. doi: 10.1186/s12874-017-0399-0.

[8] E.L. Kaplan and Paul Meier. Nonparametric estimation from incomplete observations. Journal of the American Statistical Association, 53:457-481, 1958. doi: 110.2307/2281868

[9] Jason T. Rich, J. Gail Neely, Randal C. Paniello, Courtney C. J. Voelker, Brian Nussenbaum, and Eric W. Wang. A practical guide to understanding kaplan-meier curves. Otolaryngol Head Neck Surg, 143, 2010. doi: 10.1016/j.otohns.2010.05.007

[10] Carl van Walraven and Finlay A. McAlister. Competing risk bias was common in kaplan-meier risk estimates published in prominent medical journals. J Clin Epidemiol, 69:170-3.e8, 2016. doi: 10.1016/j.jclinepi.2015.07.006.

[11] Roseanne McNamee. How serious is bias in effect estimation in randomised trials with survival data given risk heterogeneity and informative censoring? Stat Med, 36(21):3315-3333, 2017. doi: 10.1002/sim.7343.

[12] Vinay Prasad and Usama Bilal. The role of censoring on progression free survival: oncologist discretion advised. Eur J Cancer, 51(16):2269-2271, 2015. doi: 10.1016/j.ejca.2015.07.005

[13] Adrian Bagust and Sophie J Beale. Exploring the effects of early censoring and analysis of clinical trial survival data on effectiveness and cost-effectiveness estimation through a case study in advanced breast cancer. Med Decis Making, 38(7):789-796, 2018. doi: 10.1177/0272989X18790966.

[14] Zhigang Zhang and Jianguo Sun. Interval censoring. Stat Methods Med Res, 19:53-70, 2020. doi: $10.1177 / 0962280209105023$. 
[15] Kwan-Moon Leung, Robert M Elashoff, and Abdelmonem A. Afifi. Censoring issues in survival analysis. Annual Review of Public Health, 18(1):83-104, 1997. doi: 10.1146/annurev.publhealth.18.1.83.

[16] E.D. Saad and M. Buyse. Statistical controversies in clinical research: End points other than overall survival are vital for regulatory approval of anticancer agents. Annals of Oncology, 27(3):373-378, 2016. doi: 10.1093/annonc/mdv562.

[17] Rajeshwari Sridhara, Sumithra J. Mandrekar, and Lori E. Dodd. Missing data and measurement variability in assessing progression-free survival endpoint in randomized clinical trials. Clinical Cancer Research, 19(10):26132620, 2013. doi: 10.1158/1078-0432.CCR-12-2938.

[18] David Nunan, Jeffrey Aronson, and Clare Bankhead. Catalogue of bias: Attrition bias. BMJ Evidence-Based Medicine, 23(1):21-22, 2018. doi: 10.1136/ebmed-2017-110883.

[19] J. Martin Bland and Douglas G. Altman. The logrank test. BMJ, 328(7447):1073, 2004. doi: 10.1136/bmj.328.7447.1073

[20] Andre P Gabriel and Charles P Mercado. Data retention after a patient withdraws consent in clinical trials. Open Access J Clin Trials, 3:15-19, 2011. doi: 10.2147/OAJCT.S13960.

[21] Damjan Krstajic. How real is the random censorship model in medical studies? arXiv:, 2017. 1703.01237

[22] R Core Team. R: A language and environment for statistical computing. Software environment, 2018. Vienna, Austria. Version 3.5.1. https://www.R-project.org. (Last accessed 7 Dec 2020).

[23] RStudio Team. Rstudio: Integrated development environment for r. Programming languages, 2020. Boston, MA. Version 1.3.959. http://www.rstudio.com (Last accessed 7 Dec 2020.

[24] Terry M. Therneau. A package for survival analysis in r. R package, 2015. Version 2.43-3. https://CRAN. R-project.org/package=survival (Last accessed 7 Dec 2020.

[25] Mónica López-Ratón, María Xosé Rodríguez-Àlvarez, Carmen Cadarso-Suaárez, and Francisco Gude-Sampedro. Optimalcutpoints: An r package for selecting optimal cutpoints in diagnostic tests. Journal of Statistical Software, 61:1-36, 2014. doi: 10.18637/jss.v061.i08.

[26] Various Authors. Documentation for package survival version 3.2-3. Web page, 2020. https://stat.ethz ch/R-manual/R-devel/library/survival/html/00Index.html. (Last accessed 7 Dec 2020).

[27] Megan Othus, Bart Barlogie, Michael L. LeBlanc, and John J. Crowley. Cure models as a useful statistical tool for analyzing survival. Clinical cancer research, 18(14):3731-3736, 2012. doi: 10.1158/1078-0432.CCR-11-2859

[28] L. Dal Maso, S. Guzzinati, C. Buzzoni, R. Capocaccia, D. Serraino, A. Caldarella, and et al. Long-term survival, prevalence, and cure of cancer: a population-based estimation for 818902 italian patients and 26 cancer types. Ann Oncol, 25(11):2251-2260, 2014. doi: 10.1093/annonc/mdu383.

[29] Kyle Siegrist. The weibull distribution. Web page, 1997-2019. http://www.randomservices.org/random/ special/Weibull.html. (Last accessed 7 Dec 2020).

[30] Ewout W Steyerberg and Thomas A Gerds. Concepts in cancer survival analysis: research questions, data, and models. Surg Oncol, 19(2):52-4, 2010. doi.

[31] NIST/SEMATECH. Histogram interpretation: Skewed (non-normal) right. e-Handbook of Statistical Methods, 1.3.3.14.6., 2012. https://www.itl.nist.gov/div898/handbook/eda/section3/eda33e6.htm. (Last accessed 7 Dec 2020).

[32] Rupert G. Miller. Survival analysis. Book, 1997. John Wiley \& Sons. ISBN: 0-471-25218-2 https://www. wiley.com/en-es/Survival+Analysis, +2nd+Edition-p-9781118031063.

[33] L. J. Wei, D. Y. Lin, and L. Weissfeld. Regression analysis of multivariate incomplete failure time data by modeling marginal distributions. Journal of the American Statistical Association, 84:1065-1073, 1989. doi: $10.2307 / 2290084$

[34] C.L. Loprinzi, J.A. Laurie, Wieand H.S., Krook J.E., Novotny P.J., Kugler J.W., Bartel J., Law M., Bateman M., Klatt N.E., and et al. Prospective evaluation of prognostic variables from patient-completed questionnaires. north central cancer treatment group. Journal of Clinical Oncology, 12(3):601-607, 1994. doi: 10.1200/JCO.1994.12.3.601

[35] Charles G. Moertel, Thomas R. Fleming, John S. Macdonald, Daniel G. Haller, John A. Laurie, Phyllis J. Goodman, et al. Levamisole and fluorouracil for adjuvant therapy of resected colon carcinoma. New England J of Medicine, 332:352-358, 1990. doi: 10.1056/NEJM199002083220602 
[36] John H. Edmonson, Thomas R. Fleming, David G. Decker, Malkasian George D., Edward O. Jorgensen, John A. Jefferies, Maurice J. Webb, and Larry K. Kvols. Different chemotherapeutic sensitivities and host factors affecting prognosis in advanced ovarian carcinoma vs. minimal residual disease. Cancer Treatment Reports, 63:241-247, 1979. http://bit.ly/37GjmmP. (Last accessed 7 Dec 2020).

[37] John D. Kalbfleisch and Ross L. Prentice. The statistical analysis of failure time data. Wiley, New York, 1980. doi: 10.1002/9781118032985.

[38] Elie A Akl, Matthias Briel, John J You, Xin Sun, Bradley C Johnston, Jason W Busse, and et al. Potential impact on estimated treatment effects of information lost to follow-up in randomised controlled trials (lost-it): systematic review. BMJ, 344:e2809., 2012. doi: 10.1136/bmj.e2809.

[39] Birgir Gudjonsson. Survival statistics gone awry: pancreatic cancer, a case in point. J Clin Gastroenterol, 35:8180-8184, 2002. doi: 10.1097/00004836-200208000-00011.

[40] James H McMahon, Julian H Elliott, Steven Y Hong, Silvia Bertagnolio, and Michael R Jordan. Effects of physical tracing on estimates of loss to follow-up, mortality and retention in low and middle income country antiretroviral therapy programs: a systematic review. PLoS One, 8:e56047., 2013. doi: 10.1371/journal.pone.0056047.

[41] Antonio Vitor Martins Priante, Andre Lopes Carvalho, Karina de Cassia Braga Ribeiro, Hirde Contesini, and Luiz Paulo Kowalski. The importance of long-term follow-up of head and neck cancer patients for reliable survival analysis. Otolaryngol Head Neck Surg, 133:877-881, 2005. doi: 10.1016/j.otohns.2005.08.011.

[42] Kate Rosen, Vinay Prasad, and Emerson Y Chen. Censored patients in kaplan-meier plots of cancer drugs: An empirical analysis of data sharing. Eur J Cancer, 141:152-161, 2020. doi: 10.1016/j.ejca.2020.09.031.

[43] Kristin M. Sheffield, Nancy A. Dreyer, James F. Murray, Douglas E. Faries, and Megan N. Klopchin. Replication of randomized clinical trial results using real-world data: paving the way for effectiveness decisions. J Comp Eff Res, 9:1043-1050, 2020. doi: 10.2217/cer-2020-0161. 